Elsevier Editorial System(tm) for Journal of the American Society of Echocardiography

Manuscript Draft

Manuscript Number: JASE-7503R3

Title: Alterations in Cardiac Deformation, Timing of Contraction and Relaxation and Early Myocardial Fibrosis Accompany the Apparent Recovery of Acute Stress-Induced (Takotsubo) Cardiomyopathy: an End to the Concept of Transience

Article Type: Original Investigation

Keywords: Takotsubo, stress cardiomyopathy, echocardiography, strain, twist, fibrosis, T1 mapping, broken heart syndrome

Corresponding Author: Dr. Dana Dawson,

Corresponding Author's Institution: University of Aberdeen

First Author: Konstantin Schwarz, MD, MRCP, PhD

Order of Authors: Konstantin Schwarz, MD, MRCP, PhD; Trevor Ahearn, MSC, PhD; Janaki Srinivasan, RDCS; Christopher Neil, MBBS, PhD, FRACP; Caroline Scally, MBBS, MRCP; Amelia Rudd, RDCS; Baljit Jagpal, DCR(R), MSC; Michael Frenneaux, MD, FRCP, FESC, FMedSCi; Cristina Pislaru, MD, PhD; John Horowitz, MD, PhD, FRACP, FAHA; Dana Dawson

Abstract: Background: Takotsubo syndrome is an increasingly recognized cause of chest pain and occasionally of cardiogenic shock. Despite rapid improvement of the Left Ventricular Ejection Fraction (LV EF), recent registry data raises concerns about long term prognosis. We hypothesized that restoration of normal EF after acute tako-tsubo is not equivalent to full functional recovery.

Methods: We prospectively recruited 52 takotsubo patients [according to the Mayo criteria plus cardiac magnetic resonance imaging (CMR) to exclude myocardial infarction] and 44 healthy controls of the same age, gender and cardiovascular co-morbidity distribution. We focused the investigation on takotsubo patients presenting with ST-elevation type ECG or malignant arrhythmias and with LV apical ballooning variant and examined a 4 months recovery end-point. Patients underwent

Echocardiography assessment of LV myocardial deformation (Global longitudinal, radial and circumferential strain, LV twist, torsion, untwist, time to peak twist and untwist) and assessment of LV myocardial structure by pre and post contrast-enhanced CMR by T1 mapping acutely and at 4 months follow-up. Controls had a single time-point investigation. Data were analyzed using paired or unpaired tests, as appropriate for their distribution and corrected for multiple comparisons. Results: The patients' mean age was 66 years (range 28-87) and 92\% were women. All abnormal echocardiographic indices observed acutely in takotsubo patients improved (but not necessarily normalized) at followup. Significant mechano-temporal alterations characterizing both systole (global longitudinal strain, apical circumferential strain, both $\mathrm{p}<0.01$; left ventricular twist, twist rate and torsion, all $\mathrm{p}<0.0001$ ) and diastole (untwist rate and time to peak untwisting, all $p<0.001$ ) persisted at 4 months follow-up when compared with controls, despite normalization of LV ejection fraction and volumes. Whilst native T1 
(which demonstrates edema) normalized at 4 months follow-up only in

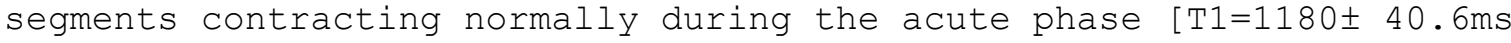
(normally contracting, $\mathrm{p}=0.2$ vs control values of $1189 \pm 16 \mathrm{~ms}$ ) and $1208 \pm$ $60.3 \mathrm{~ms}$ (dysfunctional segments, $\mathrm{p}<0.05 \mathrm{vs}$ control)], the extracellular volume fraction (ECV, which demonstrates diffuse fibrosis) remained significantly abnormal in all LV segments (whether normally contracting, $0.328 \pm 0.043, p<0.001$ or ballooning during acute presentation, $0.320 \pm 0.044, \mathrm{p}<0.001$, both vs control values of $0.273 \pm 0.045)$. Conclusion: In patients with the most clinically severe spectrum of takotsubo cardiomyopathy, regional LV systolic and diastolic deformation abnormalities persist beyond the acute event, despite normalization of global LV EF and size. In addition, although myocardial edema partly subsides, a process of global microscopic fibrosis develops in its place, detected as early as 4 months.

Suggested Reviewers:

Opposed Reviewers: 
NHS

Grampian

Dr Dana Dawson

Reader in Cardiovascular Medicine

Honorary Consultant Cardiologist

School of Medicine, Medical Sciences

and Nutrition,

University of Aberdeen,

Foresterhill

Aberdeen

Scotland

AB25 2ZD

$24^{\text {th }}$ March, 2017

Email: dana.dawson@abdn.ac.uk

Telephone: ++44 (0) 1224437969

Dear Dr Pearlman,

Thank you once again for your comments and those of the associate editors. I think it is important to get the message out to the readers in the most comprehensible manner and sometimes, after a long period of looking at the same data this becomes hard! So I am sorry about that, but thank you for your patience and for helping us make the best of it!

I hope I have addressed the 3 main points you raised in the e-mail and these are now marked in red font in the manuscript. Please let me know if you think these convey the message, if there are still confounding or unclear l'd more than happy to revise again.

Thank you once again.

With very best wishes,

Dana 


\section{Alterations in Cardiac Deformation, Timing of Contraction and Relaxation and Early Myocardial Fibrosis Accompany the Apparent Recovery of Acute Stress-Induced (Takotsubo) Cardiomyopathy: an End to the Concept of Transience}

${ }^{*}$ Konstantin Schwarz ${ }^{1}, \mathrm{MD}, \mathrm{MRCP}, \mathrm{PhD}$

*Trevor Ahearn, MSc, PhD,

Janaki Srinivasan ${ }^{1}$, RDCS

Christopher J Neil ${ }^{1}$, MBBS, PhD, FRACP

Caroline Scally, MBBS, MRCP

Amelia Rudd ${ }^{1}$, RDCS

Baljit Jagpal, DCR(R), MSc

Michael P Frenneaux ${ }^{1}$, MD, FRCP, FESC, FMedSci

Cristina Pislaru², MD, PhD

John D Horowitz ${ }^{3}$, MD, PhD, FRACP, FAHA

Dana K Dawson ${ }^{1}$, DM, FRCP, D.Phil, FESC

1. University of Aberdeen, School of Medicine and Dentistry, Aberdeen, UK

2. Mayo Clinic College of Medicine, Rochester Minnesota, USA

3. The Queen Elizabeth Hospital, Adelaide, Australia 
*the first two authors contributed equaly to this work

Word count: 4,393 excluding references, tables and figure legends

ClinicalTrials.gov Identifier: NCT02897739

This work was supported by grants from Tenovus Scotland and British Heart Foundation

There are no financial disclosures for any of the authors

Dr Dawson has a research agreement with Philips Healthcare and holds and MTA with AMAG

Pharmaceuticals

E-mail addresses:

k.schwarz@abdn.ac.uk

t.ahearn@abdn.ac.uk

j.srinivasan@nhs.net

Christopher.Neil@heartweb.com

carolinescally@abdn.ac.uk

a.e.rudd@abdn.ac.uk

baljit.jagpal@nhs.net

M.Frenneaux@uea.ac.uk

Pislaru.Cristina@mayo.edu

john.horowitz@adelaide.edu.au

dana.dawson@abdn.ac.uk

Correspondence address: Dana Dawson, School of Medicine and Dentistry, University of Aberdeen, Polwarth Building, Foresterhill, Aberdeen, AB25 2ZD, Scotland, UK

Tel: +44 1224437965

Fax: +44 1224437971

E-mail: dana.dawson@abdn.ac.uk 


\section{ABSTRACT}

Background: Takotsubo syndrome is an increasingly recognized cause of chest pain and occasionally of cardiogenic shock. Despite rapid improvement of the Left Ventricular Ejection Fraction (LV EF), recent registry data raises concerns about long term prognosis. We hypothesized that restoration of normal EF after acute tako-tsubo is not equivalent to full functional recovery.

Methods: We prospectively recruited 52 takotsubo patients [according to the Mayo criteria plus cardiac magnetic resonance imaging (CMR) to exclude myocardial infarction] and 44 healthy controls of the same age, gender and cardiovascular co-morbidity distribution. We focused the investigation on takotsubo patients presenting with ST-elevation type ECG or malignant arrhythmias and with LV apical ballooning variant and examined a 4 months recovery end-point. Patients underwent Echocardiography assessment of LV myocardial deformation (Global longitudinal, radial and circumferential strain, LV twist, torsion, untwist, time to peak twist and untwist) and assessment of LV myocardial structure by pre and post contrast-enhanced CMR by T1 mapping acutely and at 4 months follow-up. Controls had a single time-point investigation. Data were analyzed using paired or unpaired tests, as appropriate for their distribution and corrected for multiple comparisons.

Results: The patients' mean age was 66 years (range 28-87) and 92\% were women. All abnormal echocardiographic indices observed acutely in takotsubo patients improved (but not necessarily normalized) at follow-up. Significant mechano-temporal alterations characterizing both systole (global longitudinal strain, apical circumferential strain, both $p<0.01$; left ventricular twist, twist rate and torsion, all $p<0.0001$ ) and diastole (untwist rate and time to peak untwisting, all $p<0.001$ ) persisted at 4 months follow-up when compared with controls, despite normalization of LV ejection fraction and volumes. Whilst native $\mathrm{T} 1$ (which demonstrates edema) normalized at 4 months follow-up only in segments contracting normally during the acute phase $[T 1=1180 \pm 40.6 \mathrm{~ms}$ (normally contracting, $\mathrm{p}=0.2$ vs control values of $1189 \pm 16 \mathrm{~ms}$ ) and $1208 \pm 60.3 \mathrm{~ms}$ (dysfunctional 
segments, $p<0.05$ vs control)], the extracellular volume fraction (ECV, which demonstrates diffuse fibrosis) remained significantly abnormal in all LV segments (whether normally contracting, $0.328 \pm 0.043, p<0.001$ or ballooning during acute presentation, $0.320 \pm 0.044, p<0.001$, both vs control values of $0.273 \pm 0.045)$.

Conclusion: In patients with the most clinically severe spectrum of takotsubo cardiomyopathy, regional LV systolic and diastolic deformation abnormalities persist beyond the acute event, despite normalization of global LV EF and size. In addition, although myocardial edema partly subsides, a process of global microscopic fibrosis develops in its place, detected as early as 4 months. 
Keywords: Takotsubo, stress cardiomyopathy, echocardiography, strain, twist, fibrosis, T1 mapping, broken heart syndrome

\section{ABBREVIATION LIST:}

$\mathrm{BNP}=$ brain natriuretic peptide

$\mathrm{CMR}=$ cardiac magnetic resonance

$\mathrm{ECV}=$ extra cellular volume

EST = Early systolic "propping" twist

$\mathrm{IQR}=$ inter-quartile range

IVRT = isovolumic relaxation time

LGE $=$ late gadolinium enhancement

$\mathrm{LV}=$ left ventricle

$L V E F=$ left ventricular ejection fraction

$\mathrm{MI}=$ myocardial infarction

RVSP = right ventricular systolic pressure 


\section{INTRODUCTION}

Acute stress induced (takotsubo) cardiomyopathy has become much more readily recognized in recent years. Commonly masquerading an acute myocardial infarction, patients with takotsubo often have an emotional/physical trigger and have no evidence of a coronary culprit or infarct-like myocardial scar on cardiac magnetic resonance imaging ${ }^{1}$. The largest takotsubo Registry in the world as well as data form the Swedish Cardiac Catheterisation Laboratory Registry demonstrated unequivocally that the medium and long term prognosis is not benign and the mortality of takotsubo is comparable with that of myocardial infarction patients ${ }^{2,3}$. While this introduces a paradigm shift in the way that takotsubo prognosis was viewed until recently, new important questions arise, with an urgent need to explain why a condition considered until recently as "transient" and "naturally recovering" portends such a poor outlook. It has become increasingly obvious that the relatively rapid recovery of left ( \pm right) ventricular ejection fraction (LV EF) after an acute attack of takotsubo is not paralleled by symptomatic ${ }^{4}$ or ECG recovery ${ }^{5}$, for example. We have previously shown that acute takotsubo hearts demonstrate severe global edema associated with profound cardiac energetic impairment with incomplete resolution of both at 4 months ${ }^{6,7}$; additionally we showed a persistently abnormal Global Longitudinal Strain (GLS) at 3 months follow-up of a cohort of 36 tako-tsubo patients comprising all comers (ST-elevation, non-ST elevation, normal ECG and all LV-ballooning subtypes, i.e apical, mid-cavity and basal) ${ }^{4}$. However, in a condition which shows that it is on its way to recovery based on several clear objective assessments (normalized EF, decrease of myocardial oedema) there remains a disproportionately high rate of persistent symptoms, lack of return to work/physical activity, which do not appear to improve at the same rate as the objective indices. As neither edema nor energetic impairment nor an isolated reduction in GLS preclude the co-existence or recovery of a normal resting LV EF (n.b. which is different from LV performance), there must be additional patho-physiological processes that intervene, which if present in the long-term could begin to explain the unexpected poor outcome of these patients. Discrepancies in previous literature may 
have arisen due to a wide clinical spectrum of patients with takotsubo, variable follow-up, or both. The only way to further test the hypothesis of remaining uncovered pathology to explain this disproportionate persistence of symptoms relative to an apparent recovery is to focus the investigation on a homogenous and most clinically severe presenting spectrum of takotsubo patients (ST-elevation, malignant arrhythmias on presentation and large, apical-extending into mid-cavity ballooning phenotype) and to look into all (systolic and diastolic) parameters of functional non-recovery and mechanisms of potential longer-lasting changes such as myocardial fibrosis. Herewith, we describe the 4 months follow-up recovery of LV myocardial deformation and structure of a prospectively recruited cohort of takotsubo patients presenting with ST-elevation, left bundle branch block or malignant arrhythmias on ECG and with LV apical ballooning subtype.

\section{METHODS}

\section{Study Subjects}

Consecutive consenting patients who met the takotsubo Mayo Clinic $^{1}$ and currently updated ${ }^{8}$ criteria (phaeochromocytoma and myocarditis excluded also) and who, in addition, demonstrated no evidence of late gadolinium enhancement on cardiac magnetic resonance $(C M R)$ imaging were prospectively recruited at Aberdeen Royal Infirmary between 2011-2016. All patients were initially suspected of myocardial infarction and most were brought in by Scottish Ambulance Service for primary percutaneous coronary intervention. All patients had either an emotional or no clear trigger and we purposefully excluded patients with other concomitant physical triggers that could have confounded our findings: thus, patients with infections, inflammatory conditions or any other concurrent physical illnesses potentially impacting on myocardial findings in the investigators' judgement were not included in this report. All patients underwent coronary angiography and left ventriculography and had serial ECG's, 12-hour blood Troponin I, Brain natriuretic peptide (BNP), a comprehensive echocardiography and contrast-enhanced CMR within 5 days of onset of symptoms (mean time from onset of symptoms to first echo was 2 days and to first CMR was 3 
days). Left ventricular recovery was assessed with comprehensive echocardiography and CMR at 4 months follow-up (mean $122 \pm 5$ days). To account for the effect of potential confounders, a control group of 44 subjects of similar age, gender and co-morbidity distribution (matching hypertension) were recruited and underwent a single time-point echocardiography assessment. The study was approved by the North of Scotland Research Ethics Committee, ran in accordance with the Declaration of Helsinki, and all subjects provided written informed consent.

\section{Echocardiography}

A comprehensive 2D echocardiographic protocol was performed using commercially available systems [Vivid E9, equipped with a 2.5MHz (M5S) transducer (GE Vingmed, Norway] by the same operator in all subjects (JS). All measurements were taken from the first echo performed at presentation and the designated follow-up echo at 4 months. Cineloops in standard parasternal long axis, short axis, apical 4, 3 and 2-chamber views were obtained at a frame rate of at least 85

$\mathrm{Hz}$. Short axis views of the LV were taken at mitral valve level (tip of mitral valve leaflets), mid ventricular level (papillary muscle) and apical level (absence of papillary muscles). Three cardiac cycles in each view were obtained at end-expiratory breath-hold and stored for off-line analysis. Conventional spectral Doppler parameters were recorded, such as early (E) and late (A) diastolic trans-mitral flow velocities, isovolumic relaxation time (IVRT), septal and lateral mitral annulus early diastolic (E'), late diastolic (A') and systolic (S') tissue velocities.

All image analysis was performed off-line using EchoPAC software (Version 1.13, GE Healthcare) by two experienced operators (AR and JS). Left ventricular ejection fraction was calculated by the Simpson's biplane method ${ }^{9}$. Speckle-tracking strain analysis was performed after manually adjusting the automatic detection of the epicardial and endocardial borders in each view, and including all the apical myocardium (thus using an 18 segment model as opposed to the classical 17-segment one $\left.{ }^{10}\right)$. The software provided segmental and global peak longitudinal, circumferential and radial strain, from which global longitudinal peak strain (GLS) and basal, mid-cavity and apical circumferential and radial strain were calculated. LV rotations of the basal and apical levels (short 
axis views) were calculated as the average angular displacement between diastolic and systolic phases of the radial lines connecting the LV center to a specific epicardial point (Figure 1). Rotation rates were defined as peak velocities of the respective clockwise (basal) or anticlockwise (apical) rotations as viewed from the apex ${ }^{11,12}$. LV Twist $\left(^{\circ}\right)$ was defined as the net difference between the apical anticlockwise rotation and basal clockwise rotation. Torsion was defined as the twist normalized to the base-to-apex LV length measured at end-diastole $(\% / \mathrm{cm})^{13,14}$. Twist rate was calculated as the peak velocity of twist $(\% / \text { second })^{15}$. Early systolic "propping" twist (EST) was measured as the difference between the initial apical and basal rotation ${ }^{13}$. This early motion occurs during isovolumic contraction and is directed clockwise at the apex and anti-clockwise at the base (so opposite to the normal direction of movement to follow during systole, like an athlete throwing a discus who first turns to his right before spinning leftwards to throw) - this early motion is thought to have a role in "propping" the myocardium for an effective systolic twist ${ }^{13}$. Similarly, diastolic apical and basal reverse rotations were measured as the peak clockwise and anticlockwise diastolic reverse rotations of the LV short axis cross-sections, respectively. Untwist rate was determined from the rotational rate curve as the peak of the first negative deflection following the aortic valve closure as previously described ${ }^{16}$. The time to peak rotations, reverse rotations, peak twist, peak EST and peak untwist was measured from the peak of the $\mathrm{R}$ wave. In order to correct for any differences in heart rates, for all temporal analyses the R-R interval was normalized to $100 \%$. The inter-observer reproducibility of speckle-tracking LV mechanics were $5 \pm 2 \%$ (LV twist and GLS), $6 \pm 2 \%$ (EST) and 3 $\pm 1 \%$ (untwist), each performed on $n=50$ studies (20 acute Tako, 20 at 4 months follow-up and 10 controls by JS and AR). The intra-observer variability was $4 \pm 2 \%$ (LV twist and GLS), performed on all subjects included in this study (JS).

A typical LV strain curve in a normal heart is shown in Figure 2, panels A and D.

\section{Cardiac Magnetic Resonance}


A 3T Philips Achieva (Best, The Netherlands) was used for CMR imaging, the protocol included cine imaging, early and late gadolinium enhancement (Gadovist, $0.1 \mathrm{mmol} / \mathrm{kg}$ ) with swap of the phase-encoding direction and pre and post contrast Modified Look-Locker Imaging T1 mapping ${ }^{17}$. Data for T1 mapping was acquired with 3(3)3(3)5 and 5(3)3 schemes for native and post contrast T1 measurement, respectively, the latter at exactly 15 min post contrast. All CMR images were each analyzed by a pair of two independent expert observers (TA, CS, AR, CN) blinded to each other, to the other imaging modality and for T1 mapping to the order of the scan. The CMR images were analyzed in CMR Tools (Cardiovascular Imaging Solutions, London, UK) for computation of LV volumes and mass. Each segment was scored for functional status (1=normal, 2=hypokinetic, 3=akinetic, 4=dyskinetic). T1 maps were generated using Philips' RelaxMaps tool and quality controlled with goodness of fit maps (chi-squared). These were imported into Segment (Medviso, Lund, Sweden) where T1 values were output for each of the 16 segments of the 17-segment model $^{10}$ (omitting the true apex) and myocardial extra cellular volume (ECV) was calculated as previously described ${ }^{6}$. Our inter- and intra-observer variabilities for all $\mathrm{T} 1$ mapping analysis were $2.7 \pm 1.5 \%$ and $1.5 \pm 0.5 \%$ respectively and the inter-study variability for native $\mathrm{T} 1$ mapping in healthy volunteers was $2 \pm 0.5 \%$.

\section{Statistics}

Data are shown as mean \pm SD for normally distributed data, otherwise as median and interquartile range (IQR). Continuous variables were checked for normality of distribution by the Shapiro-Wilk test. Differences between acute presentation and follow-up data were examined with paired student $t$-test or Wilcoxon matched-pairs signed rank test as appropriate. Patient data at follow up was compared to that of controls using unpaired tests (unpaired $t$-test or Mann-Whitney). Categorical variables were analyzed using Chi-square test or Fisher's exact test as appropriate. In order to account for multiple comparisons the $p$ value chosen for statistical significance was Bonferroni-adjusted depending on the number of variables compared between groups after which significance was chosen at $p<0.05$. Data were analyzed using the GraphPad Prism version 5 
(GraphPad Software, San Diego California, USA) and SPSS v24. Variabilities were calculated as mean $\pm S D$ of the ratios between differences and means of the 2 independently measured variables and expressed as a percentage.

\section{RESULTS}

\section{Demographics}

Table 1 shows the patients' and controls demographics and general clinical characteristics: the majority $(79 \%)$ presented with chest pain and $87 \%$ had a stressful trigger. From the initial 52 acutely presenting patients, 44 returned for follow up. Four patients died ( 2 cardiogenic shock during acute admission, one arrhythmic cardiac arrest, one subsequent suicide) and 4 patients underwent automated implantable defibrillator/pacing device implantation (one ventricular fibrillation and long QT syndrome, one asystole after DC cardioversion for paroxysmal atrial fibrillation, and two complete heart block, one of them preceding the takotsubo acute event - these were excluded from imaging follow up as pacing is likely to affect the strain data and were unsuitable for CMR). All medication commenced for presumed acute myocardial infarction was stopped as soon as the diagnosis of takotsubo was established (i.e. after the coronary angiogram and CMR were clear-cut of diagnosis). Our center's takotsubo management policy for the duration of this enrollment has been to not extrapolate on therapies used in other conditions with different patho-physiology (i.e. myocardial infarction) in the absence of any available evidence on the basis of "primum non nocere". During their convalescence, patients continued only on the pre-existent medical therapy for any co-morbidities, except if LVEF was $<50 \%$ at discharge, in which case an angiotensin-converting enzyme inhibitor was continued empirically. The controls were of the same age and gender distribution, had similar cardiac co-morbidities and were on similar medications. Takotsubo patients had a significantly higher heart rate, both acutely and at follow-up (both $\mathrm{p}=0.001$ ), albeit their heart rate was within normal physiological limits (Tables 1 and $\mathbf{2}$ ).

\section{Strain and Twist at Presentation versus Follow up}


As shown in Table 2, there were marked abnormalities in most echocardiographic variables measured at acute presentation. Global indices of systolic and diastolic function such as LV EF and volumes as well as tissue Doppler indices of systolic (S') and diastolic (E', E/E') function were abnormal upon presentation and improved significantly at follow up (all $p<0.05$, Table 2). There were significant abnormalities at presentation compared to follow-up in LV strain (Table 3): GLS, mid-cavity radial and circumferential strain and apical radial and circumferential strain $($ all $p<0.05)$. Figure 3 shows a typical example of changes in apical circumferential strain.

Similarly, there were significant changes in the LV twist mechanics. Firstly, at presentation, $26 \%$ of takotsubo patients lacked EST usually seen in normal hearts, while this loss was less prevalent at follow up (4\%). In six (12\%) patients during the acute presentation the main systolic apical rotation was inverted (clockwise, rather than anticlockwise). Apart from one patient, the direction of the main apical rotation normalized in all patients. In those with preserved direction of apical rotation in the acute phase, the degree of rotation was reduced ( $p=0.029$ vs follow-up) and the time to peak apical rotation was also reduced ( $\mathrm{p}=0.002$ vs follow-up). Although the basal rotation was not acutely affected, the time to peak basal rotation was reduced as well $(p=0.014)$. Importantly, the magnitude of LV twist and torsion showed no significant improvement from presentation to followup, although the time to peak LV twist improved. Similarly, during diastole, the untwist rate remained severely reduced and further, the time to peak untwist remained significantly delayed, compared to controls.

\section{Strain and Twist at Follow up versus Controls}

The recovery was assessed at 4 months follow up, by comparison with controls (Tables 2 and 3). Global LV EF and end-systolic volume improved ( $p<0.0001$ by both Echo and CMR) and normalized in all but two patients. Despite the "normal LV EF", subtle persistent wall motion abnormalities remained visible in 26 patients and were all confined to the territories affected during the acute phase. These subtle wall motion abnormalities were unlike the classical reduction in wall 
thickening seen in ischemic myocardium, but were best seen as a reduction in the anticlockwise rotation of the apical short axis (which contributes to the heart's effective twist) or a slight delay in systolic contraction of some of the apical segments in long axis views. Tissue Doppler parameters (S', E') were no longer significantly different from controls although there was a trend for a delayed IVRT $(p=0.07)$ in takotsubo. LV strain indices showed a persistently impaired GLS and apical circumferential strain (both $\mathrm{p}<0.01$ ). Apical and basal rotation, their rates and time to peak all improved and were non-significantly different from controls at follow-up, however their overall contribution was not enough to restore the LV twist and torsion to normal values, which remained significantly impaired at follow-up compared to controls ( $<<0.01$ for both). The same was observed for the time to peak EST, which remained significantly prolonged in the convalescent takotsubo patients $(p=0.01)$. These systolic alterations were accompanied by residual diastolic abnormalities, i.e. reduced untwist rate and prolonged time to peak untwist rate in convalescent takotsubo compared to controls ( $p<0.01$ for both). Figure 2 shows the LV twist/untwist and rotational rates alterations seen in a typical takotsubo patient acutely (B and $\mathrm{E}$ ) and at follow up ( $\mathrm{C}$ and $\mathrm{F}$ ) compared to a normal control (A and C). Importantly, there was no significant difference in any of the systolic or diastolic strain parameters between those patients with versus those without visual wall motion abnormalities, underscoring the fact that these changes are subtle and poorly detected by the human eye beyond/above a certain threshold.

\section{Myocardial Structure by Cardiac Magnetic Resonance and T1 mapping}

CMR characteristics are shown in Table 2 and demonstrated a reduction in the LV mass at followup $(p<0.001)$. Figure 4 demonstrates that a significant increase in both native T1 and ECV values was seen acutely in both normally contracting and dysfunctional segments compared to controls: $\mathrm{T} 1=1238 \pm 64.5 / 1292 \pm 80.6 \mathrm{~ms}$ (normal/dysfunctional) vs $1189 \pm 16 \mathrm{~ms}$ (control), both $\mathrm{p}<0.001$, $E C V=0.327 \pm 0.038 / 0.326 \pm 0.040$ (normal/dysfunctional)) vs $0.273 \pm 0.045$ (control), both $p<0.001$. While native T1 normalized only in previously normally contracting segments at 4 months followup $[T 1=1180 \pm 40.6 \mathrm{~ms}$ (normal, $p=0.2$ vs control) and $1208 \pm 60.3 \mathrm{~ms}$ (dysfunctional, $p<0.05$ vs 
control)], ECV remained significantly abnormal in all LV segments (whether normally contracting, $0.328 \pm 0.043, p<0.001$ or ballooning during acute presentation, $0.320 \pm 0.044, p<0.001$, both vs control).

\section{DISCUSSION}

In this study we comprehensively assessed LV strain, twist mechanics and myocardial structure at presentation and 4 months follow-up in a homogeneously defined cohort of takotsubo patients presenting predominantly with ST-elevation type ECG or malignant arrhythmias and with apical ballooning phenotype. This cohort was prospectively recruited, is the largest of its kind undergoing this analysis to date, is matched to controls and includes male patients. The main findings of this study, incremental to previous reports, are:

1. Significant alterations in cardiac deformation, timing and rate of contraction and relaxation persisted at 4 months follow-up after takotsubo when compared with controls, despite normalization of LV ejection fraction and volumes (specifically: systole - global longitudinal strain, apical circumferential strain, LV twist, twist rate and torsion and diastole - untwist rate and time to peak untwisting).

2. After an acute takotsubo episode although myocardial edema partly subsides, a process of global microscopic fibrosis develops in its place, detected as early as 4 months.

\section{Current results in context of previous literature}

Previous studies are at variance regarding the recovery in regional LV strain at early follow-up (1 month), with one study reporting persistence of post-systolic shortening ${ }^{18}$ and another study highlighting that patients with ST-elevation type presentation may have delayed recovery especially of the diastolic function ${ }^{16}$. Both of these case-control studies found an overwhelming improvement in most parameters studied. However, in a separate, earlier cohort of patients, we documented reduced global longitudinal strain 3 months after the acute phase (a finding replicated by others at 21 days follow-up ${ }^{19}$ ), associated with incomplete resolution of symptoms in a large 
proportion of takotsubo patients ${ }^{20}$. In further separate takotsubo cohorts, we previously described additional persistent abnormalities, such as elevated natriuretic peptide concentrations at 3 months, persistent myocardial edema at 3 month $\mathrm{s}^{21}$ and impaired myocardial energetic status (together with persistence of edema) at 4 months of follow-up ${ }^{7}$. These objective abnormalities indicate that complete recovery in these patients may not occur as rapidly as previously thought and together with the persistent symptoms that we consistently observed during the clinical followup of these patients prompted us to re-evaluate cardiac mechanics. A longer convalescent period or an incomplete recovery after an acute takotsubo episode would better explain the recent registries' data which place the long-term prognosis of takotsubo at a comparable level to that of $\mathrm{MI}^{2,3,22}$. Further, the apical ballooning subtype of takotsubo studied by us here has been recently shown to have the least favorable outcome of all takotsubo variants ${ }^{23}$. Indeed, the current study, focused on the "apical ballooning" phenotype, presenting with either ST-elevation ECG, LBBB or malignant arrhythmias (thus defining a more severe clinical presenting spectrum) we demonstrate lack of recovery for several indices of systolic and diastolic performance, over and above the isolated finding of persistently abnormal GLS alone reported in previous studies inclusive of allcomers ${ }^{4}$. Therefore our study provides an important mechanistic explanation to support these large outcome studies.

This is the first study to examine the architectural recovery of the LV myocardium after a takotsubo event, using contrast-enhanced CMR. The increased LV mass ${ }^{24}$ is presumably a reflection of the significant myocardial edema seen in the myocardium during the acute stage, previously reported by us and others ${ }^{7,21,25}$. There is extensive evidence that pre-contrast $\mathrm{T} 1$ mapping is reflective of edema, previously provided from our group ${ }^{26}$ and by others ${ }^{27-29}$. Here we replicate our previous findings ${ }^{7}$ showing that LV edema subsides in the previously well contracting segments. However, despite a significant decrease by 4 months post-acute presentation, edema persists to a mild degree in the previously dysfunctional segments, where pre-contrast T1 mapping values remain significantly elevated compared to a group of age, gender and hypertensive co-morbidity controls. 
We also show that the extracellular component within the LV myocardium (expressed as a percentage of LV mass) is elevated acutely and remains at similar level at 4 months follow-up. Since the extent and severity of edema subsides during this time, it follows that something else must take its place: we believe that given the ECV values do not change, this must reflect deposition of microscopic fibrosis that develops during the convalescent phase.

\section{Unique characteristics of our cohort}

Several characteristics make the current study unique and bring added value to the currently published literature in this condition: firstly, it includes a larger number of patients (this time including male patients as well) that are of a homogeneous, most clinically severe spectrum of takotsubo presentation. Secondly, our patients were not on beta-blockers at follow-up, which is a unique cohort relative to the significant clinical equipoise present world-wide. Thirdly, our controls were matched for cardiac co-morbidities (mostly history of hypertension). The latter two characteristics eliminate important confounders in assessing LV strain and twist mechanics which are known to be affected by inotropic therapy $y^{30-34}$ and LV afterload ${ }^{35,36}$.

Importance and extent of systolic, diastolic and extracellular matrix abnormalities

The alterations in LV twist mechanics were seen both as reduced mechanical performance (reduced strain and twist) as well as significant shifts in timing of events. Firstly, the systolic changes span from a complete disappearance of the early systolic propping twist (which is a novel finding), to a reduction in LV rotation that paradoxically peaks earlier than normal, further onto a complete reversal of rotational direction. It is not clear if these alterations carry different prognostic implications. The earlier occurrence of peak rotating motion may reflect blunting of overall contraction or may represent an adaptive mechanism to the reduction in global longitudinal function (most marked during the acute phase). Importantly, these alterations result in an ineffective LV twist which remains abnormal in the convalescent phase at 4 months and matches findings of impaired longitudinal and apical circumferential strain. 
The diastolic abnormalities were equally striking: in both acute and convalescent takotsubo we found reduced untwisting rate which peaked late during diastole (on average by $20-60 \mathrm{~ms}$ compared to controls). These abnormalities are likely to lead to alterations in diastolic function and filling. Importantly, this reduced ability of the myocardium to relax persisted at 4 months follow-up, despite normalization of global functional parameters (LVEF, mitral annulus S', E', and E/E'), and is a novel finding of this study. Moreover, they make unlikely that the acute findings are due to the Q-T prolongation seen acutely in this condition, since the Q-T interval recovered in all patients at 4 months follow-up (data not shown). It is surprising therefore that classical parameters such as E', E/E' were no longer different at follow-up compared to controls. Perhaps the explanation lies in the fact that the alterations seen in takotsubo are more subtle or of a different nature compared to other cardiomyopathies. Indeed, these mechano-temporal alterations in the convalescent takotsubo hearts are present despite an apparent preservation LV geometry, since none of the patients included in this report showed any evidence of macroscopic fibrosis (scar) on CMR. Whilst these findings were observed using resting echocardiography, it is not known how these parameters would change with exercise, but it is conceivable that they provide at least a partial explanation for recent reports of fatigue and inability to engage in physical exercise in these patients ${ }^{20}$. The takotsubo patients in this study appear to sustain a relative resting tachycardia compared to controls. There are at least two possible explanations: given the mechano-temporal disruption of LV mechanics, the higher heart rate may represent a compensatory mechanism for maintaining an effective cardiac output. Another possibility is that these patients may have associated autonomic dysfunction, with parasympathetic failure, as recently suggested ${ }^{37}$, or the two conditions may co-exist. This is an important balance to establish with respect to guiding future therapies, since in one instance beta-blockers may be useful, whereas in the other they may be harmful in counteracting an adaptive mechanism.

The ECV values in themselves are comparable with those reported in other heart conditions, such as post-myocarditis, cardiomyopathies, diabetic heart and thus it is unlikely that ECV would have 
any quantitative value in differentiating the post-takotsubo heart from other conditions ${ }^{38,39}$. The real significance of our findings is the striking lack of return to a normal myocardial structure in a condition considered until recently as "a fully reversible form of acute cardiomyopathy".

Finally, our findings raise the need for longer term studies. They also may have implications for functional recovery (e.g. exercise capacity and the possibility that these patients are candidates for developing heart failure with preserved EF) and for the development of effective therapy: if a process of myocardial fibrosis persists after takotsubo then the case for specific disease-modifying therapy to prevent long term damage is strengthened. Alternatively, anti-fibrotic therapies could be considered in future studies to explore if the medium and long-term prognosis of these patients may be improved by such interventions.

\section{CONCLUSION}

We demonstrate persistence of subtle LV systolic and diastolic abnormalities at 4 months follow up in the most severe spectrum of acute stress-induced (takotsubo) cardiomyopathy, despite apparent normalization of LV EF, as compared to control subjects of similar age, gender and comorbidities distribution. In addition, although myocardial edema partly subsides, a process of global microscopic fibrosis develops in its place, detected as early as 4 months. This suggests either a longer recovery phase in this group or that this condition may not be fully reversible as previously thought. This is important in the context of therapy decision making acutely and beyond the initial period of LV EF recovery. Studies investigating potential therapies are needed. 
Data access, responsibility and analysis:

KS, TA and DD had full access to all the data in the study and take responsibility for the integrity of the data and the accuracy of the data analysis.

Acknowledgements: We would like to thank all NHS Consultant Colleagues at Aberdeen Royal Infirmary for their help with prompt recruitment of these patients (Dr M Metcalfe, Dr AD Stewart, Dr A Hannah, Dr A Noman, Dr P Broadhurst, Dr D Hogg, Dr D Garg, Dr. A Dawson) and to Margaret Bruce, RN for nurse support during the study. This work was supported by grants from Tenovus Scotland and British Heart Foundation (to Dr D Dawson, G13/10 and PG/15/108/31928 respectively). 


\section{Reference List}

1. Madhavan M, Prasad A. Proposed Mayo Clinic criteria for the diagnosis of Tako-Tsubo cardiomyopathy and long-term prognosis. Herz 2010;35(4):240-243.

2. Templin C, Ghadri JR, Diekmann J, Napp LC, Bataiosu DR, Jaguszewski M et al. Clinical Features and Outcomes of Takotsubo (Stress) Cardiomyopathy. N Engl J Med 2015;373(10):929-938 .

3. Tornvall P, Collste O, Ehrenborg E, Jarnbert-Petterson H. A Case-Control Study of Risk Markers and Mortality in Takotsubo Stress Cardiomyopathy. J Am Coll Cardiol 2016;67(16):1931-1936.

4. Neil CJ, Nguyen TH, Singh K, Raman B, Stansborough J, Dawson D et al. Relation of delayed recovery of myocardial function after takotsubo cardiomyopathy to subsequent quality of life. Am J Cardiol 2015;115(8):1085-1089.

5. Mitsuma W, Kodama M, Ito M, Tanaka K, Yanagawa T, Ikarashi N et al. Serial electrocardiographic findings in women with Takotsubo cardiomyopathy. Am J Cardiol 2007;100(1):106-109.

6. Scally C, Ahearn T, Rudd A, Neil CJ, Srivanasan J, Jagpal B et al. Right Ventricular Involvement and Recovery After Acute Stress-Induced (Tako-tsubo) Cardiomyopathy. Am J Cardiol 2016;117(5):775-780. 
7. Dawson DK, Neil CJ, Henning A, Cameron D, Jagpal B, Bruce M et al. Tako-Tsubo Cardiomyopathy: A Heart Stressed Out of Energy? JACC Cardiovasc Imaging 2015;8(8):985987.

8. Lyon AR, Bossone E, Schneider B, Sechtem U, Citro R, Underwood SR et al. Current state of knowledge on Takotsubo syndrome: a Position Statement from the Taskforce on Takotsubo Syndrome of the Heart Failure Association of the European Society of Cardiology. Eur J Heart Fail 2016;18(1):8-27.

9. Lang RM, Badano LP, Mor-Avi V, Afilalo J, Armstrong A, Ernande L et al. Recommendations for cardiac chamber quantification by echocardiography in adults: an update from the American Society of Echocardiography and the European Association of Cardiovascular Imaging. J Am Soc Echocardiogr 2015;28(1):1-39.

10. Cerqueira MD, Weissman NJ, Dilsizian V, Jacobs AK, Kaul S, Laskey WK et al. Standardized myocardial segmentation and nomenclature for tomographic imaging of the heart: a statement for healthcare professionals from the Cardiac Imaging Committee of the Council on Clinical Cardiology of the American Heart Association. Circulation 2002;105(4):539-542.

11. Notomi Y, Lysyansky P, Setser RM, Shiota T, Popovic ZB, Martin-Miklovic MG et al. Measurement of ventricular torsion by two-dimensional ultrasound speckle tracking imaging. J Am Coll Cardiol 2005;45(12):2034-2041.

12. Helle-Valle T, Crosby J, Edvardsen T, Lyseggen E, Amundsen BH, Smith HJ et al. New noninvasive method for assessment of left ventricular rotation: speckle tracking echocardiography. Circulation 2005;112(20):3149-3156. 
13. Sengupta PP, Tajik AJ, Chandrasekaran K, Khandheria BK. Twist mechanics of the left ventricle: principles and application. JACC Cardiovasc Imaging 2008;1(3):366-376.

14. Cameli M, Mondillo S, Solari M, Righini FM, Andrei V, Contaldi C et al. Echocardiographic assessment of left ventricular systolic function: from ejection fraction to torsion. Heart Fail Rev 2016;21(1):77-94.

15. Omar AMS, Vallabhajosyula S, Sengupta PP. Left Ventricular Twist and Torsion: Research Observations and Clinical Applications. Circ Cardiovasc Imaging 2015;8(6).

16. Meimoun P, Passos P, Benali T, Boulanger J, Elmkies F, Zemir H et al. Assessment of left ventricular twist mechanics in Tako-tsubo cardiomyopathy by two-dimensional speckletracking echocardiography. Eur J Echocardiogr 2011;12(12):931-939.

17. Messroghli DR, Radjenovic A, Kozerke S, Higgins DM, Sivananthan MU, Ridgway JP. Modified Look-Locker inversion recovery (MOLLI) for high-resolution T1 mapping of the heart. Magnetic resonance in medicine: official journal of the Society of Magnetic Resonance in Medicine/Society of Magnetic Resonance in Medicine 2004;52:141-146.

18. Heggemann F, Weiss C, Hamm K, Kaden J, Suselbeck T, Papavassiliu T et al. Global and regional myocardial function quantification by two-dimensional strain in Takotsubo cardiomyopathy. Eur J Echocardiogr 2009;10(6):760-764.

19. Kim SA, Jo SH, Park KH, Kim HS, Han SJ, Park WJ. Functional recovery of regional myocardial deformation in patients with takotsubo cardiomyopathy. J Cardiol 2016. 
20. Neil CJ, Nguyen TH, Singh K, Raman B, Stansborough J, Dawson D et al. Relation of delayed recovery of myocardial function after takotsubo cardiomyopathy to subsequent quality of life. Am J Cardiol 2015;115(8):1085-1089.

21. Neil C, Nguyen TH, Kucia A, Crouch B, Sverdlov A, Chirkov Y et al. Slowly resolving global myocardial inflammation/oedema in Tako-Tsubo cardiomyopathy: evidence from T2-weighted cardiac MRI. Heart 2012;98(17):1278-1284.

22. Stiermaier T, Moeller C, Oehler K, Desch S, Graf T, Eitel C et al. Long-term excess mortality in takotsubo cardiomyopathy: predictors, causes and clinical consequences. Eur J Heart Fail 2016.

23. Ghadri JR, Cammann VL, Napp L. Differences in the Clinical Profile and Outcomes of Typical and Atypical Takotsubo Syndrome. JAMA Cardiol 2016;1(3):335-340.

24. Eitel I, Von Knobelsdorff-Brenkenhoff F, Bernhardt P, Carbone I, Muellerleile K, Aldrovandi A et al. Clinical characteristics and cardiovascular magnetic resonance findings in stress (takotsubo) cardiomyopathy. JAMA - Journal of the American Medical Association 2011;306:277-286.

25. Eitel I, Lucke C, Grothoff M, Sareban M, Schuler G, Thiele H et al. Inflammation in takotsubo cardiomyopathy: insights from cardiovascular magnetic resonance imaging. European Radiology 2010;20:422-431.

26. Cameron D, Siddiqi N, Neil CJ, Jagpal B, Bruce M, Higgins DM et al. T(1) mapping for assessment of myocardial injury and microvascular obstruction at one week post myocardial infarction. Eur J Radiol 2016;85(1):279-285. 
27. Dall'Armellina E, Piechnik S, Ferreira V, Si QL, Robson M, Francis J et al. Cardiovascular magnetic resonance by non contrast T1-mapping allows assessment of severity of injury in acute myocardial infarction. Journal of Cardiovascular Magnetic Resonance 2012;14(1):15.

28. Ferreira V, Piechnik S, Dall'Armellina E, Karamitsos T, Francis J, Choudhury R et al. Noncontrast T1-mapping detects acute myocardial edema with high diagnostic accuracy: a comparison to T2-weighted cardiovascular magnetic resonance. Journal of Cardiovascular Magnetic Resonance 2012;14(1):42.

29. Dall'Armellina E, Karia N, Lindsay AC, Karamitsos TD, Ferreira V, Robson MD et al. Dynamic Changes of Edema and Late Gadolinium Enhancement after Acute Myocardial Infarction and Their Relationship to Functional Recovery and Salvage Index. Circ Cardiovasc Imaging 2011;4:228-236.

30. Takemoto Y, Hozumi T, Sugioka K, Takagi Y, Matsumura Y, Yoshiyama M et al. Beta-blocker therapy induces ventricular resynchronization in dilated cardiomyopathy with narrow QRS complex. J Am Coll Cardiol 2007;49(7):778-783.

31. Leong DP, Chakrabarty A, Shipp N, Molaee P, Madsen PL, Joerg L et al. Effects of myocardial fibrosis and ventricular dyssynchrony on response to therapy in new-presentation idiopathic dilated cardiomyopathy: insights from cardiovascular magnetic resonance and echocardiography. Eur Heart J 2012;33(5):640-648.

32. Blondheim DS, Kazatsker M, Friedman Z, Lysyansky P, Meisel SR, Asif A et al. Effect of medical therapy for heart failure on segmental myocardial function in patients with ischemic cardiomyopathy. Am J Cardiol 2007;99(12):1741-1744. 
33. Tanaka H, Matsumoto K, Sawa T, Miyoshi T, Motoji Y, Imanishi J et al. Evaluation of global circumferential strain as prognostic marker after administration of beta-blockers for dilated cardiomyopathy. Int J Cardiovasc Imaging 2014;30(7):1279-1287.

34. Mizuguchi $\mathrm{Y}$, Oishi $\mathrm{Y}$, Miyoshi $\mathrm{H}$, luchi A, Nagase N, Ara N et al. Possible mechanisms of left ventricular torsion evaluated by cardioreparative effects of telmisartan in patients with hypertension. Eur J Echocardiogr 2010;11(8):690-697 .

35. Balmain B, Stewart GM, Yamada A, Chan J, Haseler LJ, Sabapathy S. The impact of an experimentally induced increase in arterial blood pressure on left ventricular twist mechanics. Exp Physiol 2016;101(1):124-134.

36. Weiner RB, Weyman AE, Kim JH, Wang TJ, Picard MH, Baggish AL. The impact of isometric handgrip testing on left ventricular twist mechanics. J Physiol 2012;590(20):5141-5150.

37. Norcliffe-Kaufmann L, Kaufmann H, Martinez J, Katz SD, Tully L, Reynolds HR. Autonomic Findings in Takotsubo Cardiomyopathy. Am J Cardiol 2016;117(2):206-213.

38. Radunski UK, Lund GK, Saring D, Bohnen S, Stehning C, Schnackenburg B et al. T1 and T2 mapping cardiovascular magnetic resonance imaging techniques reveal unapparent myocardial injury in patients with myocarditis. Clin Res Cardiol 2016.

39. Wong TC, Piehler KM, Kang IA, Kadakkal A, Kellman P, Schwartzman DS et al. Myocardial extracellular volume fraction quantified by cardiovascular magnetic resonance is increased in diabetes and associated with mortality and incident heart failure admission. Eur Heart $\mathrm{J}$ 2014;35(10):657-664. 


\section{FIGURE TITLES and LEGENDS}

Figure 1: Example of basal rotation $(\mathrm{Br})$ and Apical rotation $(\mathrm{Ar})$; black arrow depicting the direction of motion; blue interrupted dash = end-diastolic position, blue full dash = peak-systolic position; the software calculated angular displacement is plotted against time within a cardiac cycle and shown in Figure 2.

Figure 2: Examples of Twist curves $\left(^{\circ}\right)(\mathrm{A}-\mathrm{C})$ and Rotational rate curves [Twist rate and untwist rate $(\% /$ second)] (D-F)

A: matched control volunteer, showing a characteristic early systolic twist (EST) in a clockwise rotation at the apex (blue trace) and in an anticlockwise rotation at the base (purple trace), occurring during the iso-volumic contraction. This is followed by the anticlockwise apical rotation ( $A r$, blue) and clockwise basal rotation (Br, purple) which result in the net systolic twist during LV ejection (Twist, white line).

B: Takotsubo patient at presentation demonstrating a near disappearance of the EST, complete reversal in the apical rotation and reduction in overall LV twist;

C: Takotsubo patient at 4 months follow up showing incomplete recovery of LV twist

D: matched control volunteer, E: Takotsubo patient at presentation, F: Takotsubo patient at follow up; Both $\mathrm{E}$ and $\mathrm{F}$ show lack of recovery of twist and untwist rates.

Figure 3: Apical circumferential strain

A: Takotsubo patient at presentation, B: the same patient at follow up, C: healthy control Red color indicates more negative strain value (circumferential shortening during systole), blue color indicates positive strain (circumferential lengthening during systole, in this case associated with apical ballooning in takotsubo patient)

Figure 4: Microscopic fibrosis in acute and recovered Takotsubo cardiomyopathy.

Native T1 and ECV in dysfunctional and normally contracting segments compared to controls acutely and at follow-up. $*=p<0.001$ acute vs controls, $\dagger=p<0.05$ follow-up vs control 
Click here to download high resolution image

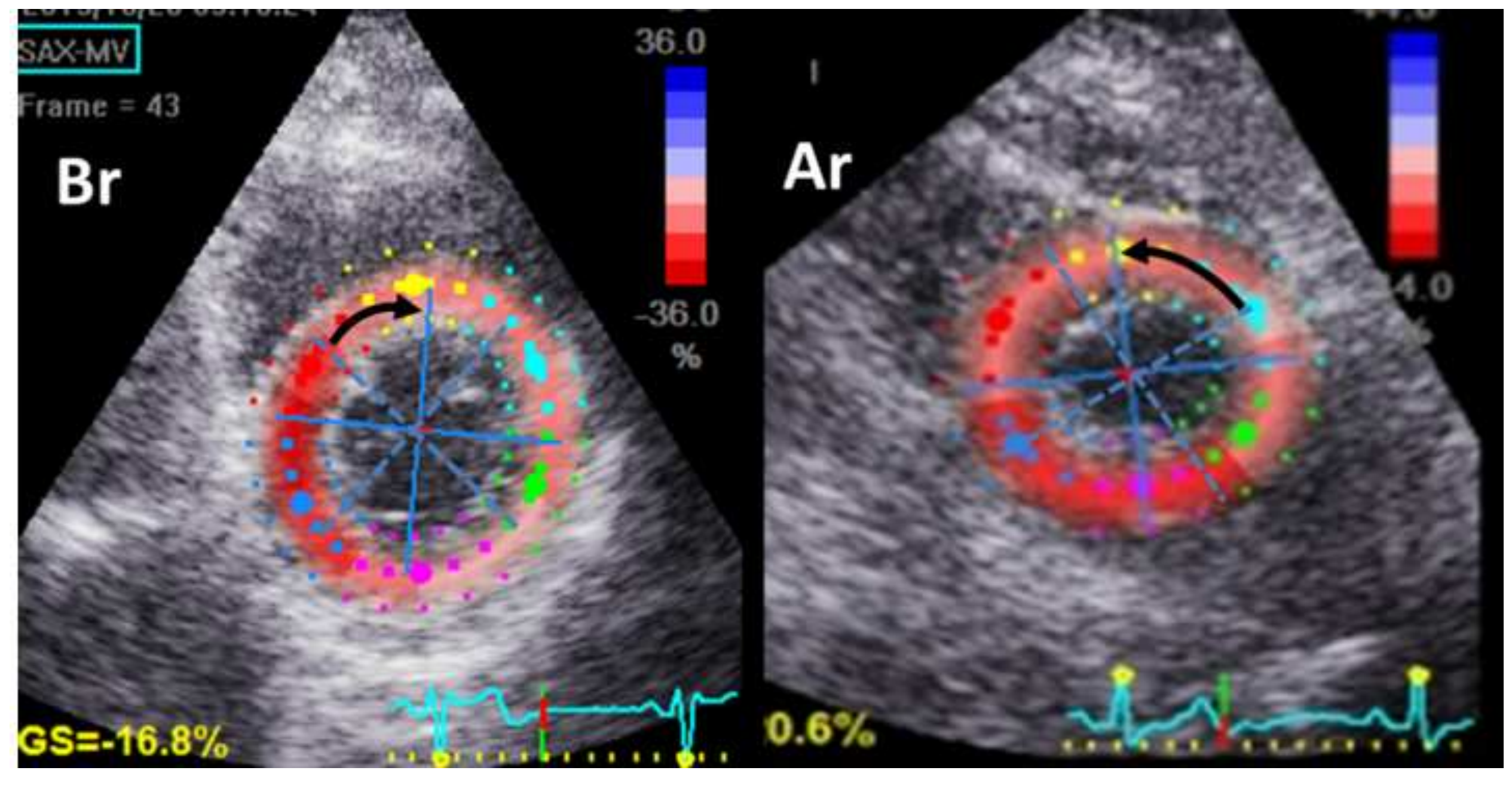

\section{Click here to download high resolution image}



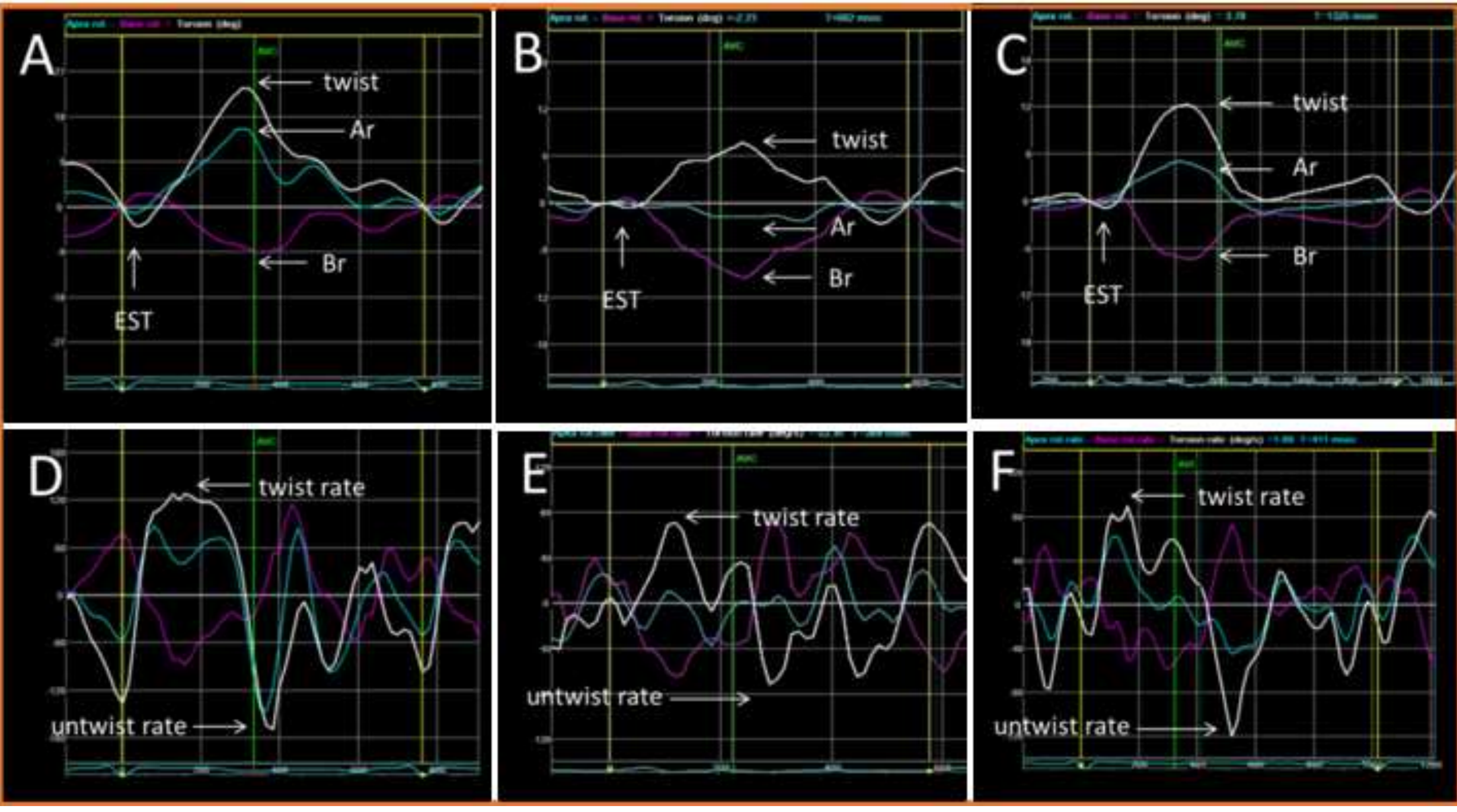
Figure 3
Click here to download high resolution image

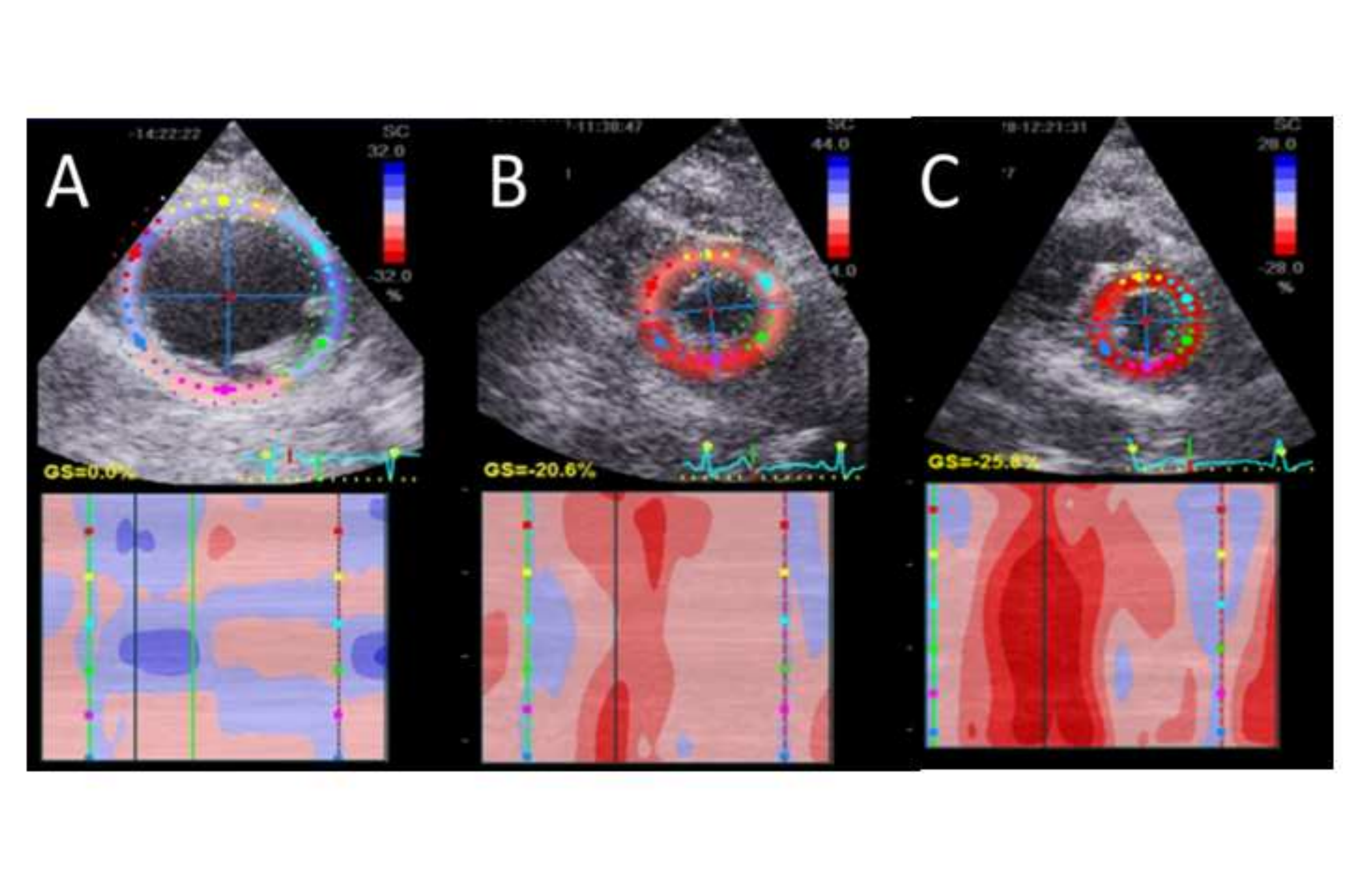

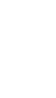

.
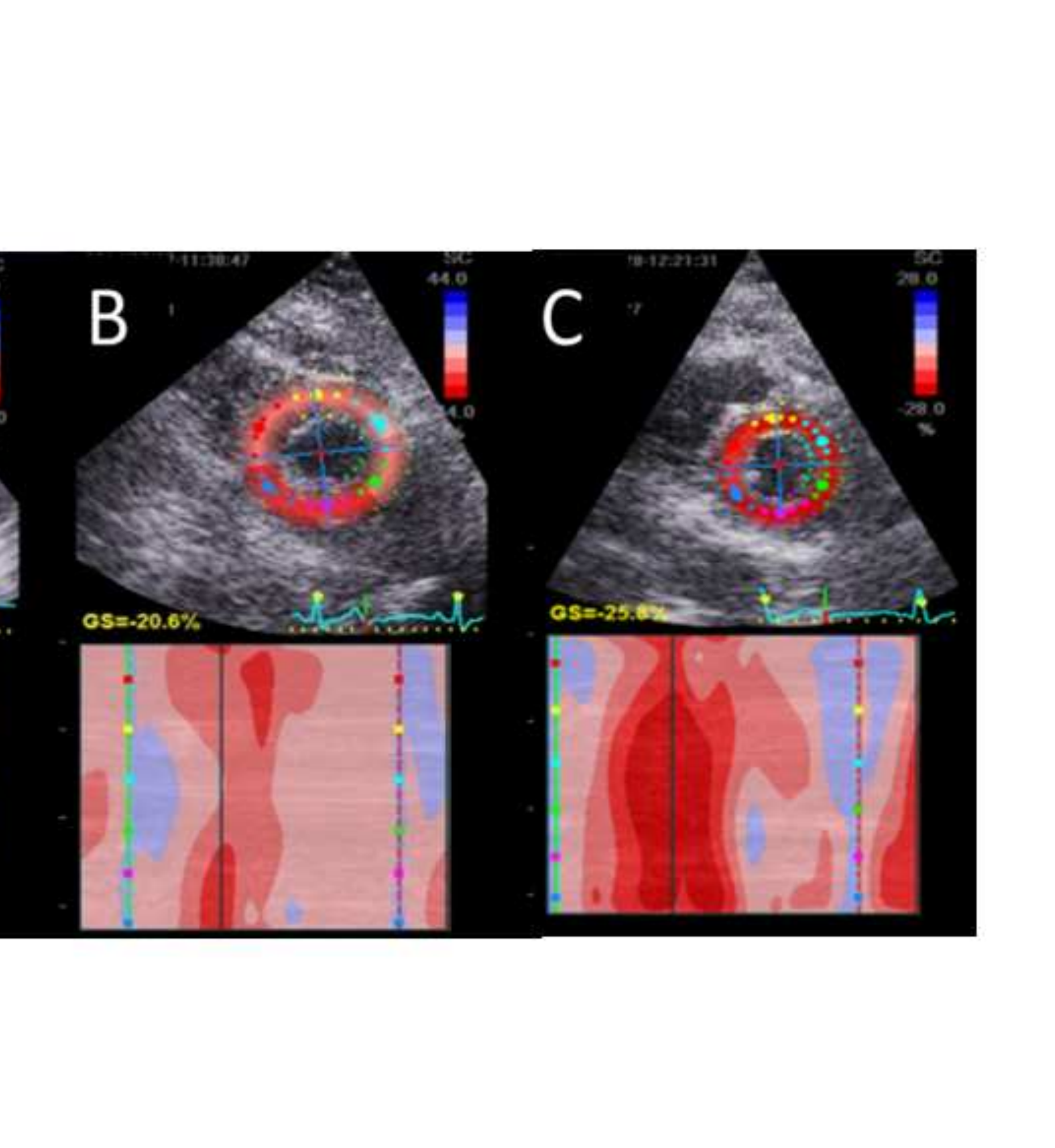
Figure 4

Click here to download high resolution image

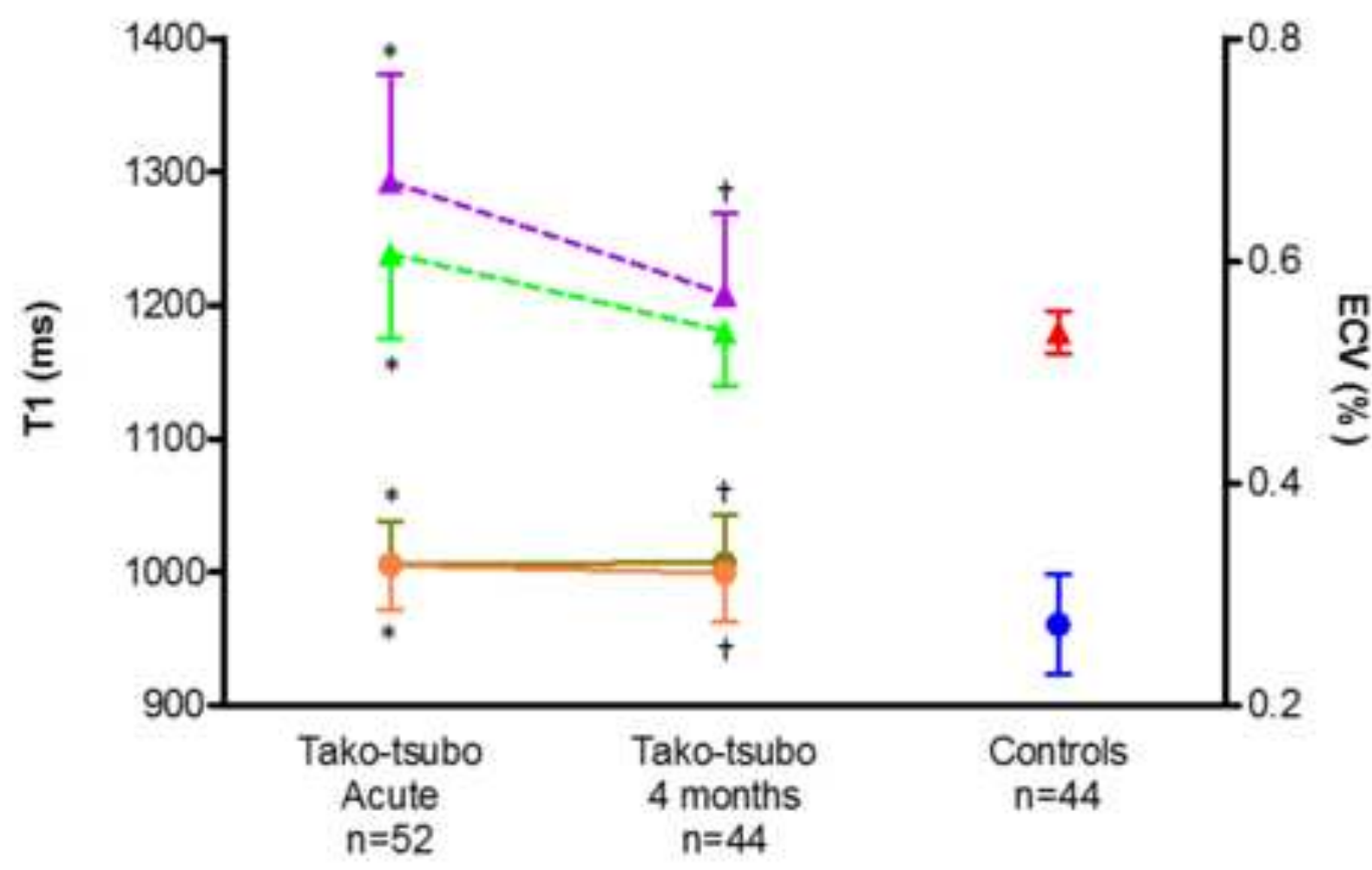

- * T1 Dysfunctional segments

-A. T1 Normal segments

A T1 Controls

$\stackrel{\text { m }}{2}$

- ECV Dysfunctional segments

- ECV Normal segments

- ECV Controls

$\begin{array}{ccc}\text { Tako-tsubo } & \text { Tako-tsubo } & \text { Controls } \\ \substack{\text { Acute } \\ n=52} & 4 \text { months } & n=44 \\ n=44 & \end{array}$


Table 1: Clinical characteristics of patients and controls

\begin{tabular}{|c|c|c|c|c|}
\hline Demographics & $\begin{array}{l}\text { Tako-tsubo } \\
\text { acute } \\
\text { presentation } \\
\qquad(n=52)\end{array}$ & $\begin{array}{l}\text { Tako-tsubo } \\
\text { follow up } \\
(n=44)\end{array}$ & $\begin{array}{l}\text { Controls } \\
(n=44)\end{array}$ & $\begin{array}{c}\text { p } \\
\text { follow-up } \\
\text { vs } \\
\text { Controls }\end{array}$ \\
\hline Age (years) & $66 \pm 11$ & $65 \pm 10$ & $67 \pm 10$ & 0.418 \\
\hline Female, n (\%) & 47 (92\%) & 40 (90\%) & $40(90 \%)$ & 1.000 \\
\hline BMI & $26 \pm 4$ & $26 \pm 5$ & $25 \pm 2$ & 0.423 \\
\hline \multicolumn{5}{|l|}{ Past medical History } \\
\hline Hypertension & $15(28 \%)$ & $10(23 \%)$ & $12(28 \%)$ & 0.771 \\
\hline "Angina" & $2(4 \%)$ & 0 & & $\mathrm{n} / \mathrm{a}$ \\
\hline Diabetes & $5(9 \%)$ & $4(9 \%)$ & $3(8 \%)$ & 0.238 \\
\hline Thyroid disease & $13(26 \%)$ & $11(27 \%)$ & 0 & $0.004^{*}$ \\
\hline Mental health disease & $17(34 \%)$ & $14(32 \%)$ & 0 & $0.024^{*}$ \\
\hline Smoker /Exsmoker & $2(4 \%) / 6(11 \%)$ & $2(4 \%) / 6(11 \%)$ & $1(3 \%) / 7(18 \%)$ & 0.338 \\
\hline Alcohol & $6(11 \%)$ & $5(11 \%)$ & $6(13 \%)$ & 0.722 \\
\hline \multicolumn{5}{|l|}{ Medication n (\%) } \\
\hline Aspirin & $6(11 \%)$ & $5(11 \%)$ & $6(13 \%)$ & 1.000 \\
\hline Beta-blocker & $5(9 \%)$ & $3(7 \%)$ & $4(10 \%)$ & 0.238 \\
\hline$A C E-I / A R B$ & $14(27 \%)$ & $10(23 \%)$ & $6(13 \%)$ & 0.084 \\
\hline$C C B$ & $4(8 \%)$ & $3(7 \%)$ & $3(8 \%)$ & 1.000 \\
\hline Heart rate & $80 \pm 16$ & $81 \pm 16$ & $67 \pm 10$ & $0.001^{*}$ \\
\hline Systolic BP, mmHg & $127 \pm 23$ & $125 \pm 23$ & $129 \pm 17$ & 0.113 \\
\hline Diastolic BP, mmHg & $76 \pm 16$ & $76 \pm 16$ & $76 \pm 10$ & 0.977 \\
\hline \multicolumn{5}{|l|}{ Presentation (n) } \\
\hline Chest pain & $41(79 \%)$ & & & \\
\hline Pre-/syncope & $9(17 \%)$ & & & \\
\hline Pulmonary oedema & $2(4 \%)$ & & & \\
\hline \multicolumn{5}{|l|}{ Presenting ECG, n (\%) } \\
\hline ST elevation & $41(79 \%)$ & & & \\
\hline
\end{tabular}


$\angle B B B$

Arrhythmia

(VT/VF/CHB/asystole)

\section{Troponin I}

Admission

12 hours

CRP (mg/L)

BNP - presentation $(\mathrm{pg} / \mathrm{mL})$

BNP - follow-up (pg/mL)

Coronary atheroma $(<50 \%)$

LV-angiogram type, n (\%)

apical

$52(100 \%)$

$5(10 \%)$

$6(11 \%)$

$586 \pm 720$

$48 \pm 26$

$15(28 \%)$
$1.56(0.5-3.7)$

$3.81(1.2-8.8)$

$5.5(<0.04$ to 14.8$)$

${ }^{*}=p<0.05$ after multiple comparisons corrections

Abbreviations: $\mathrm{ACE}=$ angiotensin converting enzyme, $\mathrm{ARB}=$ angiotensin receptor blocker, $\mathrm{BMI}=\mathrm{body}$ mass index, $\mathrm{BP}=$ blood pressure, $\mathrm{CRP}=\mathrm{C}$-reactive protein, $\mathrm{BNP}=$ Brain natriuretic peptide 
Table 2: 2D Echocardiography and basic CMR variables

\begin{tabular}{|c|c|c|c|c|c|}
\hline Echocardiography characteristics & $\begin{array}{c}\text { Tako-tsubo acute } \\
\text { presentation } \\
n=52\end{array}$ & $\begin{array}{c}\text { Tako-tsubo } \\
\text { follow up } \\
n=44\end{array}$ & $\begin{array}{c}p \text { value } \\
\text { (acute vs } \\
\text { follow-up, paired } \\
\text { analysis) }\end{array}$ & $\begin{array}{c}\text { Controls } \\
n=44\end{array}$ & $\begin{array}{c}p \text { value } \\
\text { (follow-up vs } \\
\text { controls, unpaired } \\
\text { analysis) }\end{array}$ \\
\hline$E F, \%$ & $45 \pm 13$ & $62 \pm 9$ & $<0.0001^{*}$ & $64 \pm 6$ & 0.342 \\
\hline LV EDV, ml & $82(71-102)$ & $71(61-86)$ & $0.006^{*}$ & $67 \pm 14$ & 0.138 \\
\hline LV ESV, ml & $44(33-66)$ & $28(20-33)$ & $0.0001^{*}$ & $23(21-27)$ & 0.047 \\
\hline $\mathrm{SV}, \mathrm{ml}$ & $39 \pm 14$ & $45 \pm 12$ & $0.042^{*}$ & $42 \pm 9$ & 0.396 \\
\hline Mod/Severe MR, n & $4 / 0$ & $1 / 0$ & 0.643 & $0 / 0$ & 1.000 \\
\hline LVOT Vmax , m/s & $0.9 \pm 0.2$ & $0.9 \pm 0.2$ & 0.357 & $1.0 \pm 0.1$ & 0.361 \\
\hline $\mathrm{AV} V \max , \mathrm{m} / \mathrm{s}$ & $1.3 \pm 0.3$ & $1.4 \pm 0.3$ & 0.130 & $1.4 \pm 0.2$ & 0.572 \\
\hline $\mathrm{E}, \mathrm{m} / \mathrm{s}$ & $0.7 \pm 0.2$ & $0.7 \pm 0.1$ & 0.363 & $0.7 \pm 0.1$ & 0.708 \\
\hline $\mathrm{A}, \mathrm{m} / \mathrm{s}$ & $0.7 \pm 0.1$ & $0.7 \pm 0.2$ & 0.312 & $0.7 \pm 0.1$ & 0.959 \\
\hline$E / A$ & $0.8(0.6-1.1)$ & $0.8(0.7-1.2)$ & 0.435 & $0.9(0.7-1.2)$ & 0.544 \\
\hline IVRT, ms & $121 \pm 34$ & $114 \pm 32$ & 0.164 & $100 \pm 21$ & 0.072 \\
\hline $\mathrm{E}^{\prime}, \mathrm{cm} / \mathrm{s}$ & $5 \pm 2$ & $7 \pm 2$ & $0.0002^{*}$ & $8 \pm 2$ & 0.179 \\
\hline $\mathrm{S}^{\prime}, \mathrm{cm} / \mathrm{s}$ & $5(4-6)$ & $7(5-8)$ & $0.002^{*}$ & $7(6-8)$ & 0.499 \\
\hline$E / E^{\prime}$ & $11(9-15)$ & $9(7-12)$ & $0.020^{*}$ & $8(7-11)$ & 0.310 \\
\hline
\end{tabular}


TR Vmax, m/s

Estimated RVSP, $\mathrm{mmHg}$

CMR characteristics

LVEDV, ml

LVESV, ml

$E F, \%$

LVM, g

LVEDVi, $\mathrm{ml} / \mathrm{m}^{2}$

LVESVi, $\mathrm{ml} / \mathrm{m}^{2}$

LVMi, $\mathrm{g} / \mathrm{m}^{2}$
$2.6 \pm 0.6$

$35(25-45)$

$132(110-152)$

$56(46-83)$

$53(47-62)$

$76(65-85)$

$34(24-47)$

$80 \pm 15 \dagger$
139 (114-154)
$2.4 \pm 0.3$

$30 \pm 8$

$44(36-54)$

$64(60-68)$

$118(109-133)$

$70(63-83)$

$26(20-32)$

$68(57-78)$
$126(112-141)$

0.170

$2.3 \pm 0.3$

0.265

0.057

$27 \pm 6$

0.149

${ }^{*}=\mathrm{p}<0.05$ after multiple comparisons corrections $\begin{array}{lll}0.214 & 118 \pm 16 & 0.126\end{array}$

$\begin{array}{lll}0.000^{*} & 41 \pm 9 & 0.308\end{array}$

$\begin{array}{lll}0.000 * & 66 \pm 4 & 0.956\end{array}$

$\begin{array}{lll}0.001^{*} & 106 \pm 21 & 0.070\end{array}$

$0.206 \quad 69 \pm 7 \quad 0.938$

$\begin{array}{lll}0.000 * & 24 \pm 5 & 0.187\end{array}$

$0.001^{*} \quad 61 \pm 10$

Abbreviations: LV EDV=left ventricular end-diastolic volume, LVEDVi= left ventricular end-diastolic volume index, LV ESV=left ventricular end-systolic volume, LVESVi= left ventricular end-systolic volume index, LVM=left ventricular mass, LVMi=left ventricular mass index. 
Table 3: LV Twist mechanics

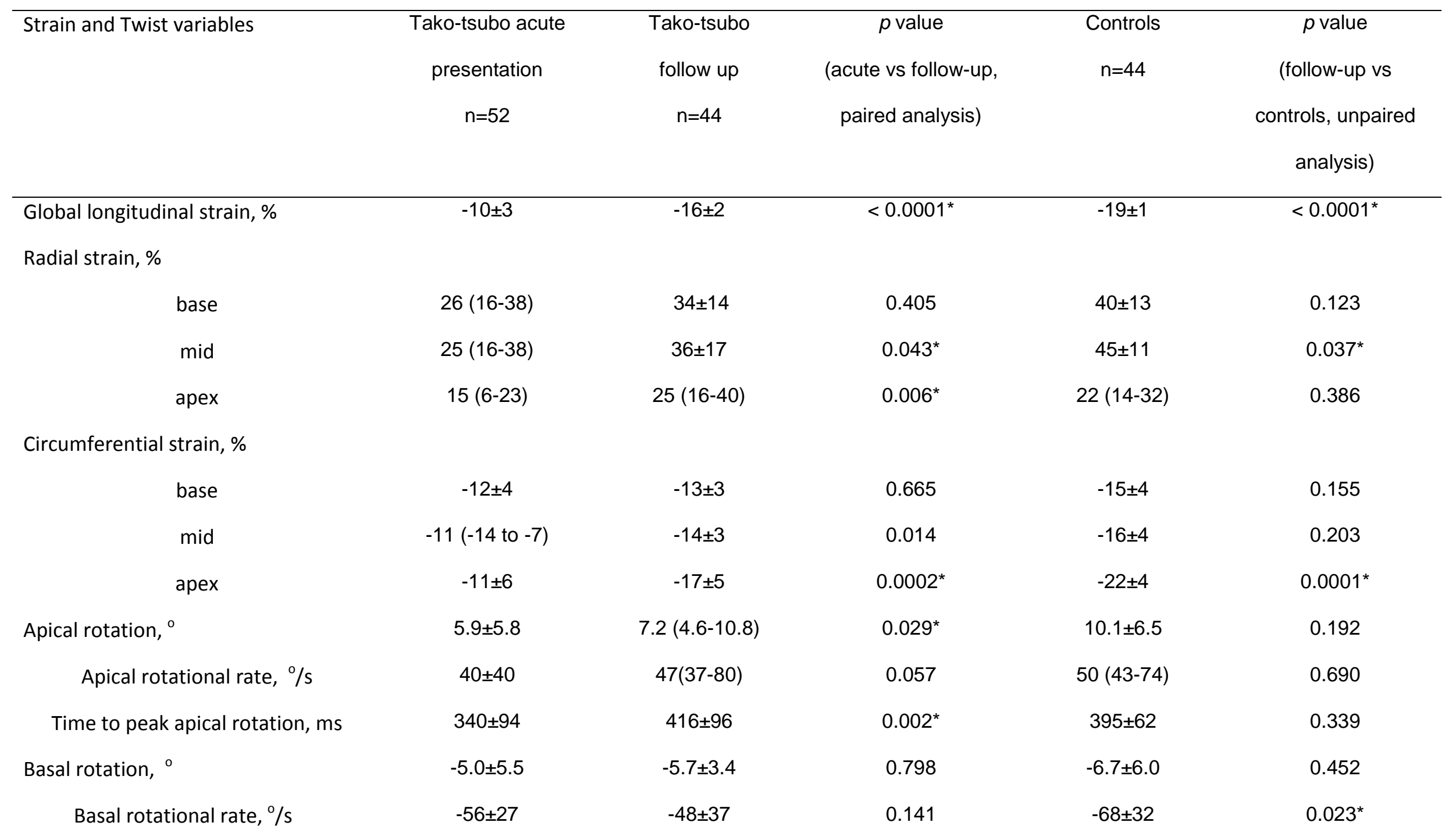


Time to peak basal rotation, $\mathrm{ms}$

LV Twist,

Time to peak LV twist, ms

LV Torsion, $\%$ cm

LV twist rate, $\%$

Early systolic twist (EST), ${ }^{\circ}$

Missing EST

Time to peak EST, ms

Untwist rate, $\% / \mathrm{s}$

Time to peak untwist rate, $\mathrm{ms}$

\begin{tabular}{|c|c|c|c|c|}
\hline $331 \pm 95$ & $404 \pm 84$ & $0.014^{*}$ & $384 \pm 96$ & 0.216 \\
\hline $11 \pm 7$ & $12 \pm 6$ & 0.298 & $22 \pm 4$ & $<0.0001^{*}$ \\
\hline $343 \pm 80$ & $404 \pm 72$ & $0.001^{*}$ & $384 \pm 47$ & 0.244 \\
\hline $1.4 \pm 0.9$ & $1.7 \pm 0.9$ & 0.243 & $3.1 \pm 0.8$ & $<0.0001^{*}$ \\
\hline $80 \pm 38$ & $80 \pm 35$ & 0.747 & $115 \pm 23$ & $<0.0001^{*}$ \\
\hline $0.7(-1.8$ to 0.0$)$ & $-1.5 \pm 2.3$ & 0.255 & $-2.1 \pm 1.3$ & 0.252 \\
\hline $8(25.8 \%)$ & $1(3.8 \%)$ & 0.070 & 0 & 1.000 \\
\hline $62(38-102)$ & $75 \pm 34$ & 0.868 & $53 \pm 26$ & $0.011^{*}$ \\
\hline$-76 \pm 33$ & $-77 \pm 34$ & 0.824 & $-118 \pm 27$ & $<0.0001^{*}$ \\
\hline $505 \pm 104$ & $545 \pm 76$ & 0.182 & $484 \pm 79$ & $0.006^{*}$ \\
\hline
\end{tabular}

${ }^{*}=\mathrm{p}<0.05$ after multiple comparisons corrections

Data is shown as mean $\pm S D$ if normally distributed or median (IQR) if non-parametric. 
Copyright Transfer Letter

(1)

\begin{abstract}
This piece of the submission is being sent via mail.

Thanster
\end{abstract}

(1)

aranteris 